Review Article

\title{
The Effect of Chinese Herbal Medicine Gualouxiebaibanxia Decoction for the Treatment of Angina Pectoris: A Systematic Review
}

\author{
Wei Liu, ${ }^{1}$ Xingjiang Xiong, ${ }^{2}$ Xiaochen Yang, ${ }^{2}$ Fuyong Chu, ${ }^{1}$ and Hongxu Liu ${ }^{1}$ \\ ${ }^{1}$ Department of Cardiology, Beijing Hospital of Traditional Chinese Medicine, Capital Medical University, Beijing 100010, China \\ ${ }^{2}$ Department of Cardiology, Guang'anmen Hospital, China Academy of Chinese Medical Sciences, Beijing 100053, China
}

Correspondence should be addressed to Fuyong Chu; chufuyong2014@163.com and Hongxu Liu; lhxcardiology@163.com

Received 3 April 2016; Accepted 8 August 2016

Academic Editor: Giuseppe Caminiti

Copyright (C) 2016 Wei Liu et al. This is an open access article distributed under the Creative Commons Attribution License, which permits unrestricted use, distribution, and reproduction in any medium, provided the original work is properly cited.

\begin{abstract}
We systematically assess the current clinical evidence of Gualouxiebaibanxia (GLXBBX) decoction for the treatment of angina pectoris (AP). We included RCTs testing GLXBBX against conventional drugs and GLXBBX combined with conventional drugs versus conventional drugs. 19 RCTs involving 1730 patients were finally identified, and the methodological quality was evaluated as generally low. The results of the meta-analysis showed that GLXBBX alone had significant effect on improving angina symptoms (RR: 1.24, 95\% CI 1.14 to 1.35; $P<0.00001$ ), ECG (RR: 1.28 [1.13, 1.44]; $P<0.0001$ ), and HDL-C (MD: 0.56 [0.54, 0.58$] ; P<0.00001$ ) compared with anti-arrhythmic drugs. A significant improvement in angina symptoms (RR: 1.17 [1.12, 1.22]; $P<0.00001)$ and ECG $(\mathrm{RR}=1.22 ; 95 \% \mathrm{CI}=[1.14,1.30] ; P<0.00001)$ was observed for GLXBBX plus conventional drugs when compared with conventional drugs. Eight trials reported adverse events without serious adverse effects. GLXBBX appears to have beneficial effects on improvement of ECG and reduction of angina symptoms in participants with AP. However, the evidence remains weak due to the poor methodological quality of the included studies. More rigorous trials are needed to confirm the results.
\end{abstract}

\section{Introduction}

Coronary artery disease (CAD) is one of the main causes of morbidity and mortality worldwide [1-3]. CAD was the most common cause of death globally in 2013, resulting in 8.14 million deaths (16.8\%) up from 5.74 million deaths (12\%) in 1990 [4]. Coronary heart disease burden is projected to rise from around 47 million disability-adjusted life years (DALYs) globally in 1990 to 82 million DALYs in 2020 [5]. Nearly $58 \%$ of patients with coronary artery disease were suffering from chronic stable angina [6]. In every one million people in the general population of most European countries, it is estimated that 20,000 to 40,000 individuals suffer from angina pectoris $(\mathrm{AP})[7,8]$.

Angina significantly limits the ordinary activities of most of these patients and worsens their quality of life (QOL), in terms of not only physical activity/pain but also mental health $[9,10]$. Current treatment strategies aim to reduce the risk of mortality and morbid events, reduce symptoms, and improve QOL $[3,11]$. Despite multiple treatment options including pharmacotherapy (as organic nitrates, $\beta$-blockers, and calcium channel antagonists), revascularization, lifestyle changes, and aggressive management of modifiable coronary artery disease risk factors [11, 12], a high proportion of patients with stable angina remains symptomatic and widely used antianginal therapies have side effects, including headache, postural hypotension, and dizziness, and the continuous uptake of nitrates may lead to drug tolerance $[13,14]$. Perhaps, there has been a growing interest in alternative therapies for AP.

Gualouxiebaibanxia (GLXBBX) decoction, which originated in the Eastern Han Dynasty (25-220), is a traditional Chinese medicinal herbal containing four commonly used herbs (Trichosanthes kirilowii Maxim., Allium macrostemon, and white spirit). It has long been used to treat chest pain in clinical practice in China. Its mechanism of action could be related to promoting Qi to activate blood and removing blood stasis, activating Yang, and eliminating phlegm based 
on the theory of Traditional Chinese Medicine (TCM). Recent studies revealed that GLXBBX could dilate coronary arteries, antiplatelet aggregative activity, and antioxidant and hypolipidemic effect and ameliorates myocardial fibrosis [1518]. Currently, there have been numbers of research works indicating that GLXBBX is effective to AP, whereas the data supporting the validity is not enough. This systematic review aims to assess the current clinical evidence for the efficacy of GLXBBX for the treatment of AP by integrating different outcome measures from randomized controlled trials (RCTs).

\section{Material and Methods}

2.1. Information Sources and Search Strategies. A systematic search was conducted in the following 7 online electronic databases from their inception until January 20, 2016: Cochrane Central Register of Controlled Trials (CENTRAL, 1996-2016), PubMed (1959-2016), EMBASE (19802016), Chinese Scientific Journal Database (VIP, 1989-2016), Chinese Biomedical Literature Database (1978-2016), Wanfang database (WMOD, 1985-2016), and Chinese National Knowledge Infrastructure (CNKI, 1979-2016). In addition, as GLXBBX is mainly prescribed in China, we also conducted a literature search of the website of the Chinese Clinical Trial Registry (available at http://www.chictr.org.cn/index.aspx); the reference lists of retrieved articles were also reviewed. No restriction on publication status or language was imposed. Search terms used were ("angina pectoris" OR "coronary artery disease") AND ("herb" OR "Gualouxiebaibanxia Decoction" OR "GuaLouXieBaiBanXia” OR "Gualouxiebaibanxia Tang") AND ("randomized controlled trial” OR "controlled clinical trial” OR "random” OR "randomly" OR "randomized” OR “control”).

2.2. Eligibility Criteria. Studies that met the following criteria were included: (1) GLXBBX combined with conventional drugs versus conventional drugs; (2) GLXBBX versus conventional drugs; and (3) duration of treatment being at least 2 weeks. The participants who were suffering from and had been diagnosed with AP should be included regardless of the severity. The primary outcome measures in RCTs were mortality due to ischemic heart disease or incidence of heart events; the secondary outcome measure was reduction of angina symptoms (RAS) and ECG improvements. Other outcomes like frequency of angina attack (FAA), blood lipid, follow-up, and adverse events were also measured. Duplicated publications reporting the same groups of participants were excluded. The clinical standards of AP are by "the International Society and Federation of Cardiology/World Health Organization (ISFC/WHO) [38]" or "ACC/AHA 2002 guideline update for the management of patients with chronic stable angina task force on practice guidelines (2002 ACCF/AHA) [39]." Effective symptomatic improvements should achieve at least $50 \%$ (basic) or $80 \%$ (significant) reduction of angina symptoms (RAS) [38]. Effective ECG improvements should achieve at least $0.05 \mathrm{mV}$ lowering at ST segment in ECG (basic) or nearly normal (significant) ECG during an exercise test [38].
2.3. Study Selection and Data Collection Process. Two reviewers (Wei Liu and Xingjiang Xiong) independently screened the titles, abstracts, and key words of each searched article according to the eligibility criteria. One author (Xiaochen Yang) extracted data from the included RCTs and then put them into Microsoft Excel. Another 2 authors (Wei Liu and Fuyong Chu) examined the accuracy of extracted data. The data extraction form comprised the authors, title, publication year, sample size, age, sex distribution, diagnosis standard, study design, interventions in the treatment and control groups, composition of GLXBBX or modified GLXBBX, trial duration, outcome measures, and adverse effects. If there were discrepancies in the process of selection, whether to include or exclude a study was resolved by a third author (Hongxu Liu).

2.4. Quality Assessment. The methodological quality of trials was assessed independently by two authors (Xiaochen Yang and Xingjiang Xiong) using criteria from the Cochrane Handbook for Systematic Review of Interventions [40]. The qualities of included RCTs were assessed by six specific domains, including random sequence generation, allocation concealment, blinding of participants and personnel, blinding of outcome data, incomplete outcome data, and selective reporting. We judged each item from three levels ("yes" for a low risk of bias, "no" for a high risk of bias, and "unclear" otherwise). Then the methodological quality of the trials was ranked into three levels: low risk of bias (all items with low risk of bias), high risk of bias (at least one item with high risk of bias), or unclear risk of bias (at least one item with an unclear domain).

2.5. Statistical Analysis. Meta-analyses of RCTs were performed by using RevMan 5.1 software (The Nordic Cochrane Centre, The Cochrane Collaboration, Copenhagen, 2011). Dichotomous data were expressed as relative risk (RR) or continuous outcomes as weighted mean difference (WMD), both with 95\% confidence intervals (CI). Subgroups analysis was conducted among different types of comparisons (including GLXBBX versus conventional drugs and GLXBBX plus conventional drugs versus conventional drugs). Heterogeneity between trials was recognized as significant when $I^{2}>50 \%$ or $P<0.1$. The fixed effects model was used to analyze data with low heterogeneity (heterogeneity test, $P>0.10$ ), whereas the random effects model was applied if heterogeneity was significant (heterogeneity test, $P<0.10$ ). Publication bias was assessed by funnel plot analysis if the group included more than 10 trials [40].

\section{Results}

3.1. Study Identification. An initial screening yielded 418 potentially relevant citations in accordance with the search strategy. A total of 176 articles were screened after 242 duplicates of the same articles included in different databases were removed. According to the inclusion criteria, 145 articles were excluded on the basis of the titles and abstracts. These studies were primarily excluded because they were not RCTs. 


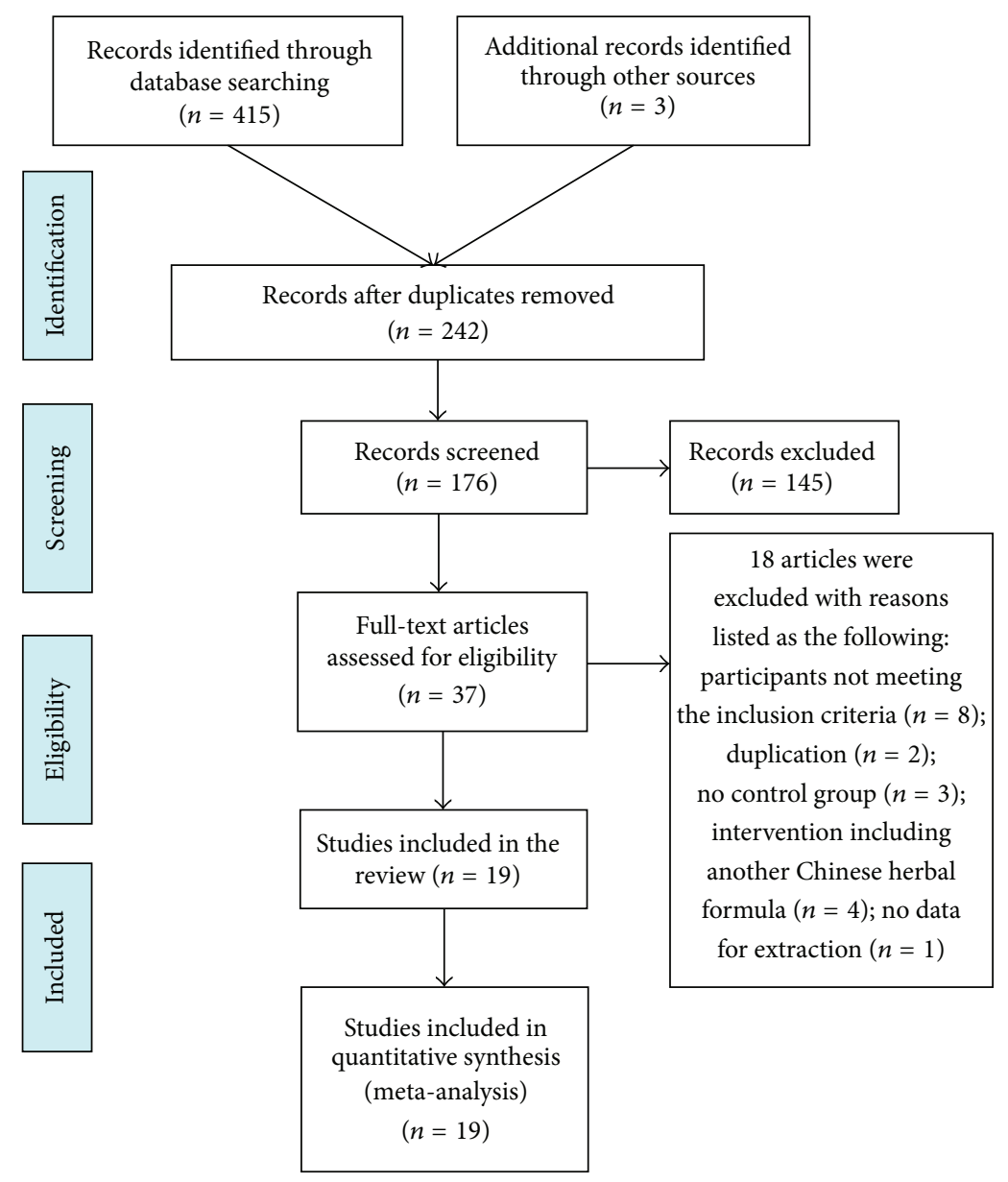

FIgURE 1: Flow diagram of study selection and identification.

A total of 37 full-text articles were retrieved for further assessment, of which 18 were excluded for the following reasons: participants not meeting the inclusion criteria $(n=$ $8)$; duplication $(n=2)$; no control group $(n=3)$; intervention including another Chinese herbal formula $(n=4)$; and no data for extraction $(n=1)$. In the end, 19 RCTs were included, and all trials had been conducted and published in China. A flow chart depicted the search process and study selection (as shown in Figure 1).

3.2. Study Characteristics. The 19 RCTs [19-37], which involved a total number of 1730 patients with angina pectoris, ranging from 60 to 210, were published between 2001 and 2015. The age of the angina pectoris patients ranged from 35 to 90 years. The duration of treatment varied from 2 weeks to 8 weeks. The dosage of GLXBBX was one dose twice a day. Three diagnostic criteria of AP were specified: six trials [19, $25,27,29,32,37]$ used the Guidelines of Clinical Research of New Drugs of Traditional Chinese Medicine (GCRNDTCM); ten trials $[20-22,24,28,30,31,33,34,36]$ used the International Society and Federation of Cardiology/World Health Organization-1979 (1979 ISFC/WHO); one trial [35] used "ACCF/AHA Guideline for the Diagnosis and Management of Patients with Unstable Ischemic Heart Disease-2002 (2002
ACCF/AHA). There were two comparisons: 6 trials [19-24] compared GLXBBX and conventional drugs alone and 13 trials [25-37] compared the combination of GLXBBX and conventional drugs with conventional drugs. Reductions in angina symptoms and improvement in ECG were the most commonly measured outcomes in the included studies. The other outcomes included changes of blood lipid and frequency of angina attack. Eight trials reported adverse events $[19,24,25,27,33,35-37]$. The descriptive information of the included trials and subjects in this review was summarized in Table 1 and GLXBBX's dosages and compositions are listed in Table 2.

3.3. Study Quality. Among trials, only 9 studies [21, 24, 26, $32,33,35-37]$ stated the method of the sequence generation with random number table and drawing [20], while none of the 19 studies reported details for sample size calculations and none was double-blind, placebo controlled study. Additionally, none mentioned allocation concealment or blinding methods. None reported information on follow-up and two trials $[33,35]$ had reported dropout without explaining their reasons. Among all RCTs, the characteristics of participants in each study arm were similar at baseline (age, race, sex, and disease course). Selective reporting could not be evaluated 


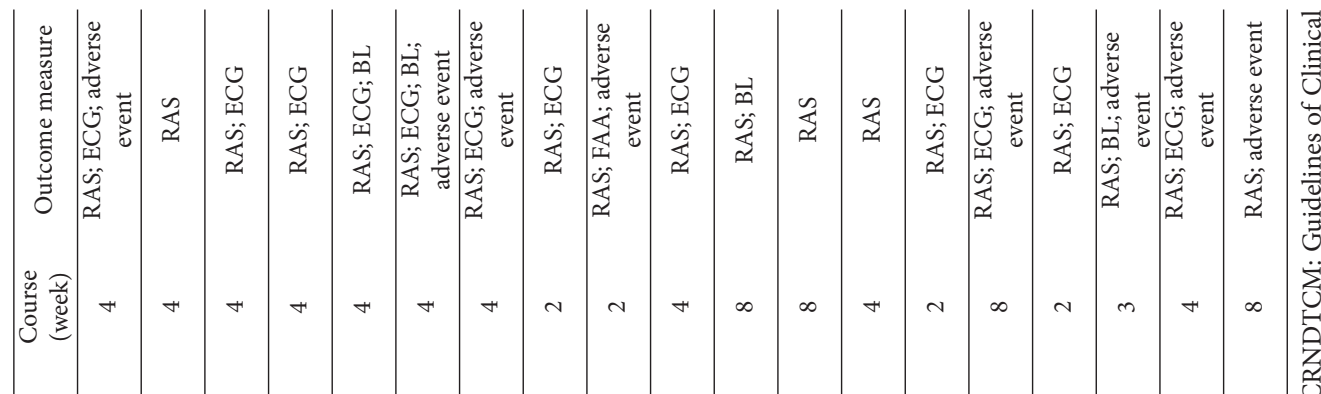

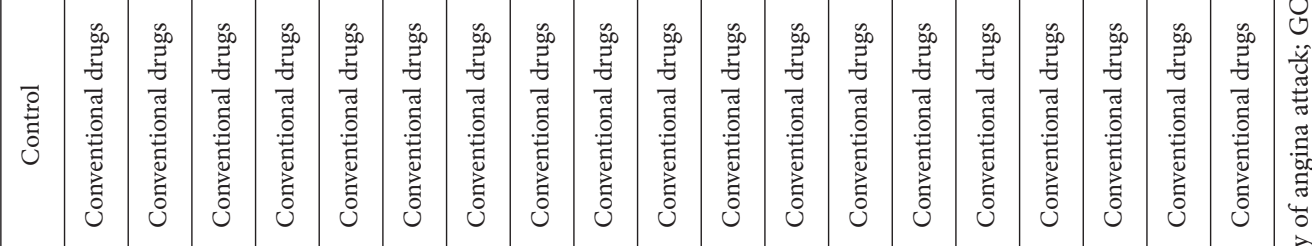

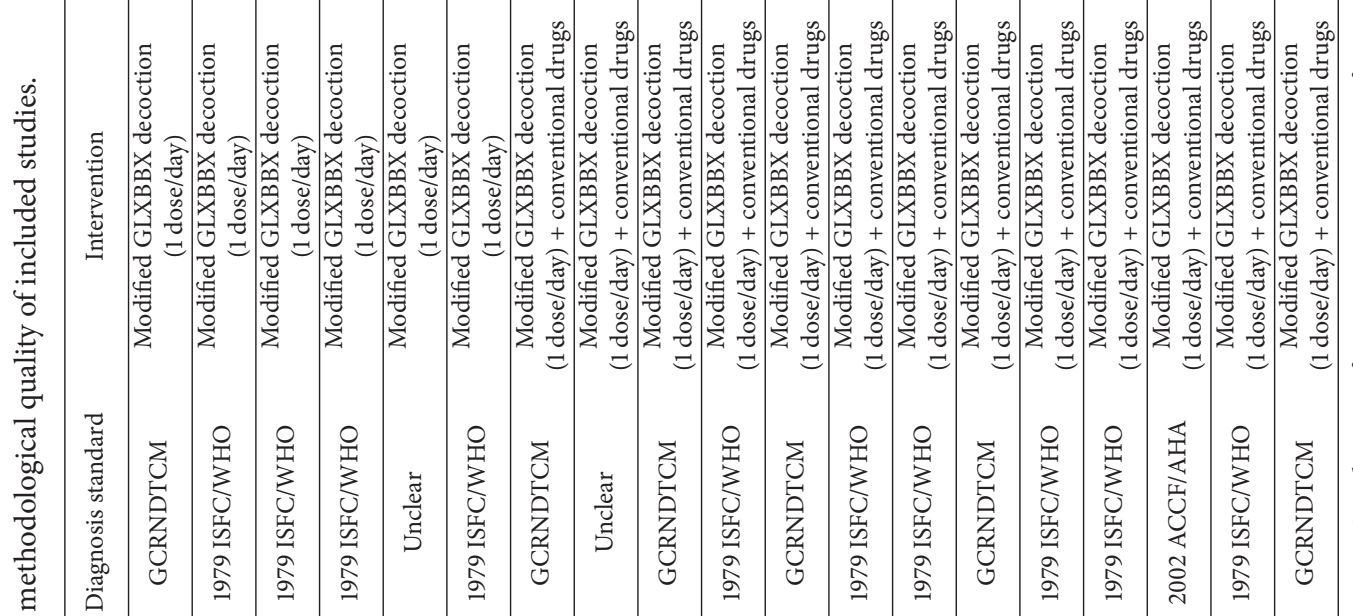

莺

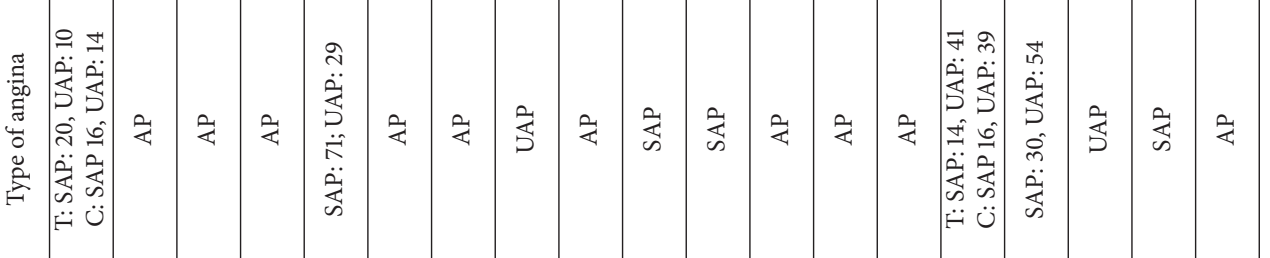

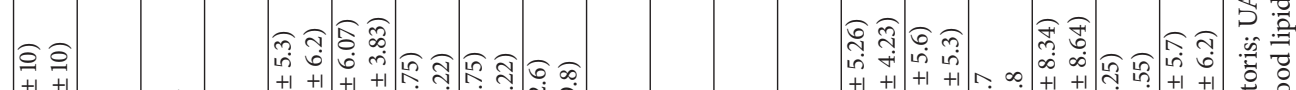

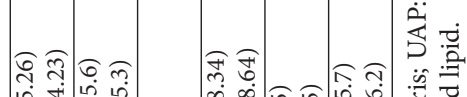

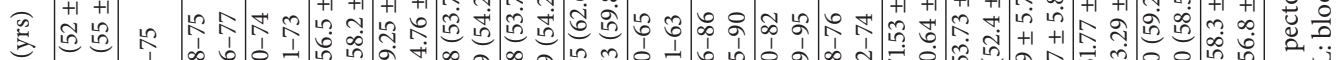

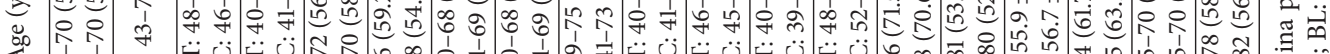

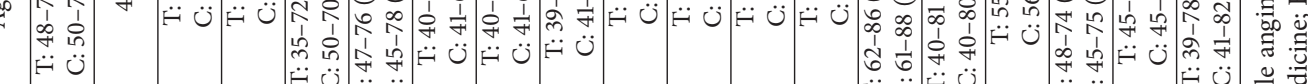

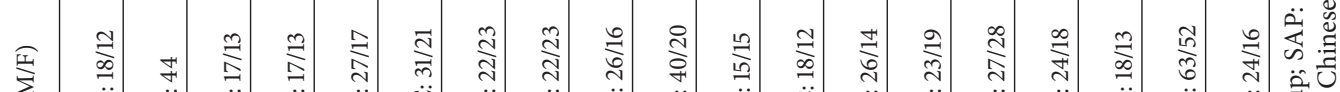

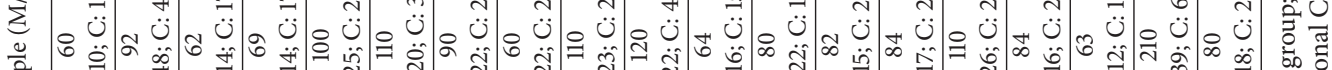

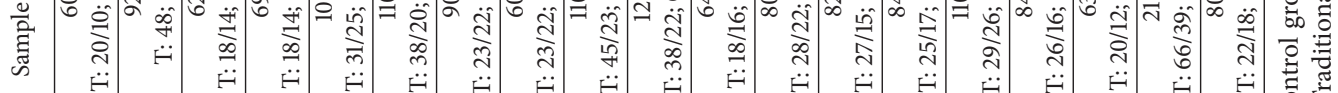

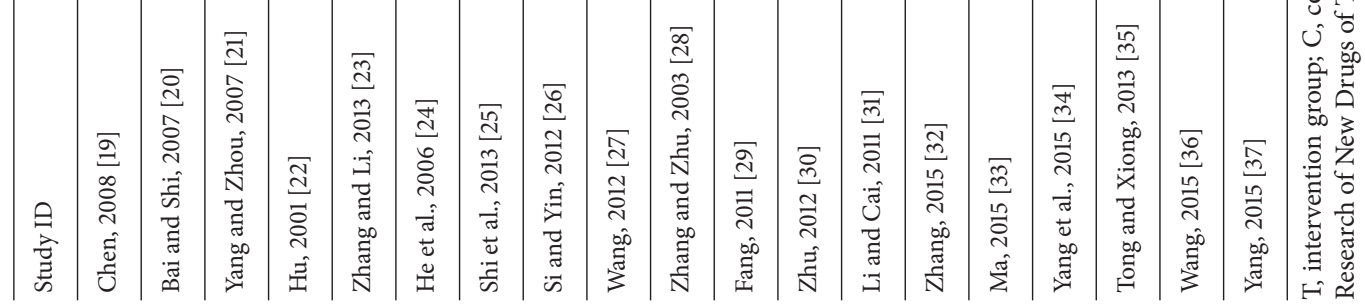




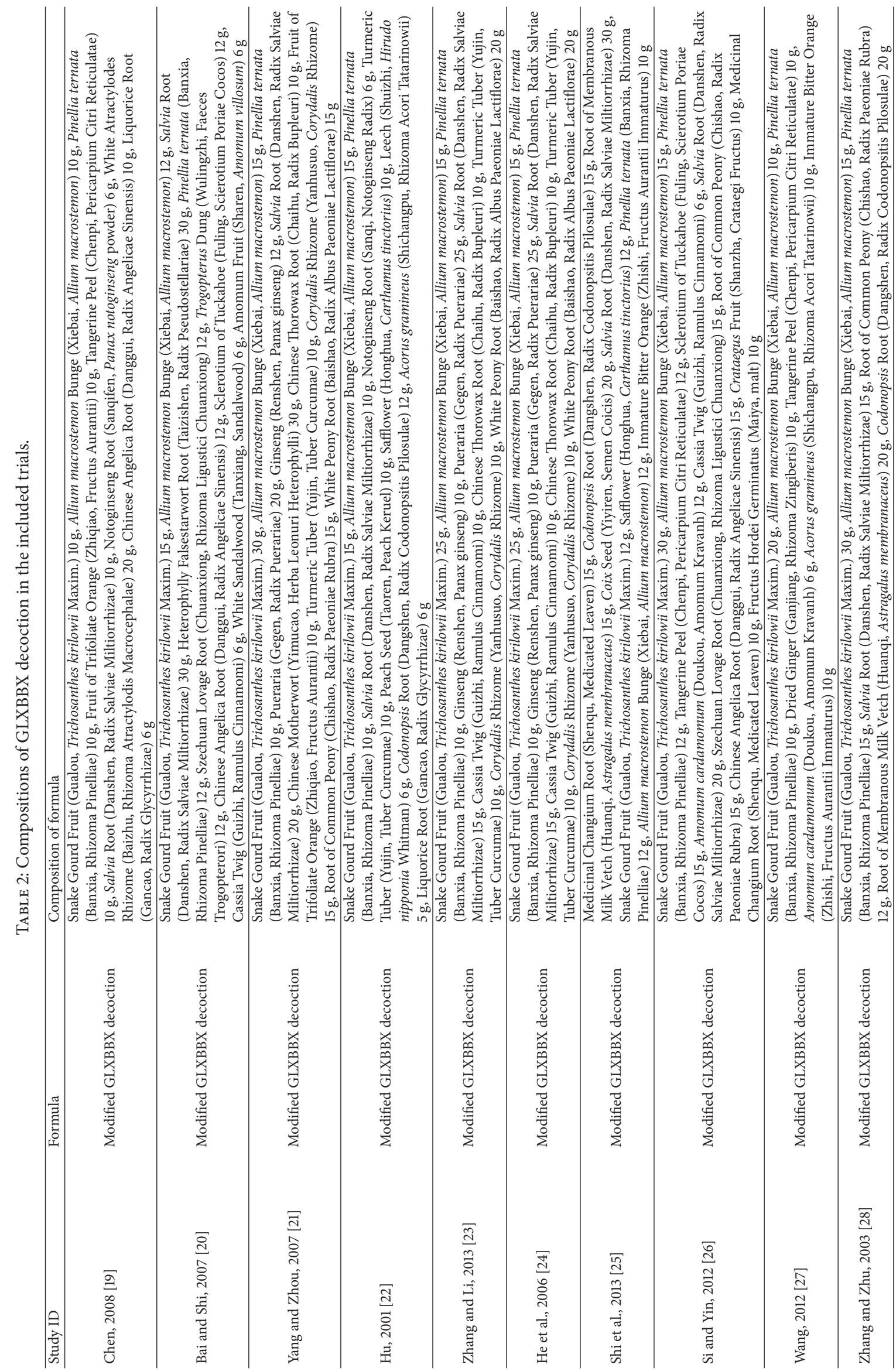




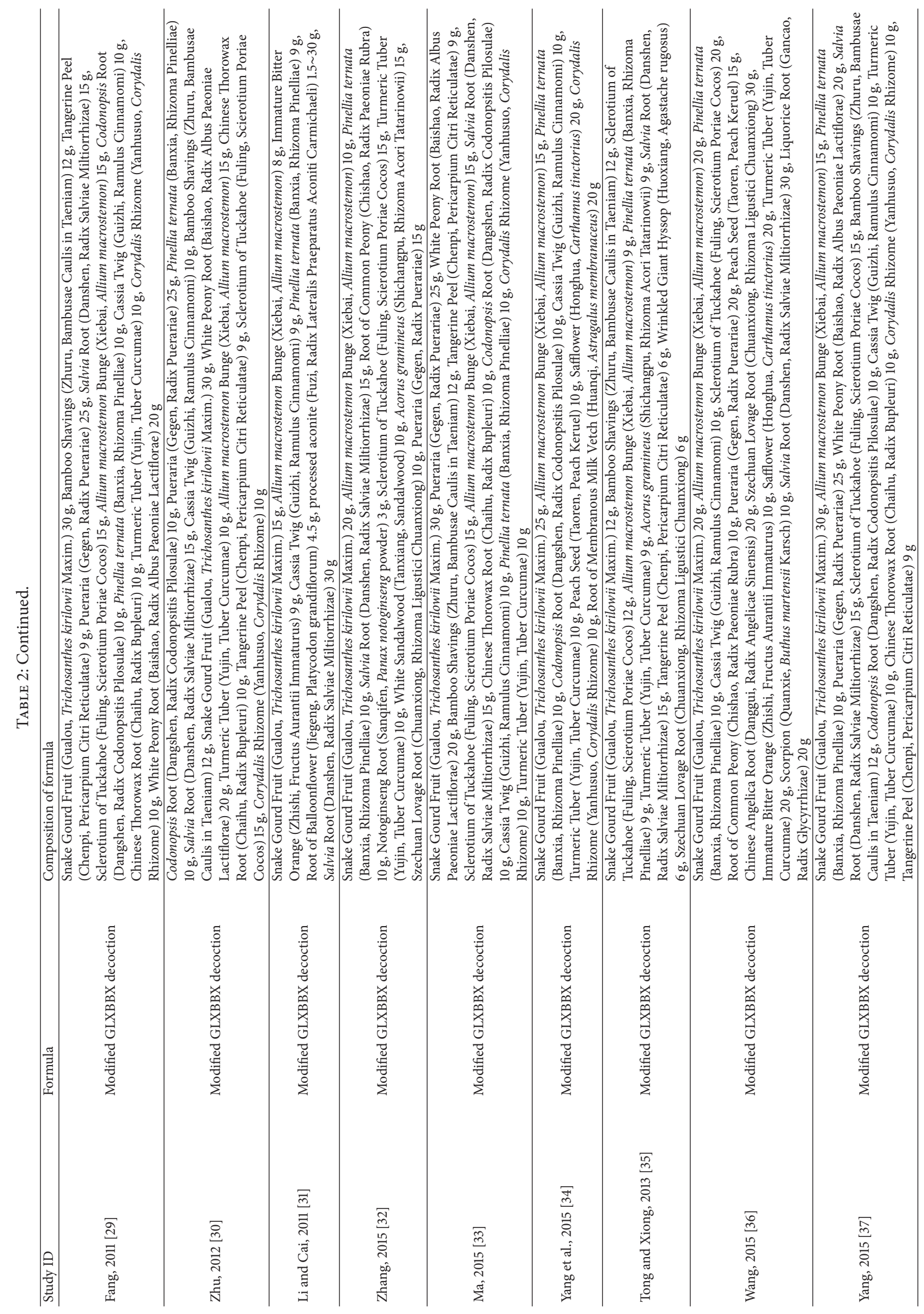




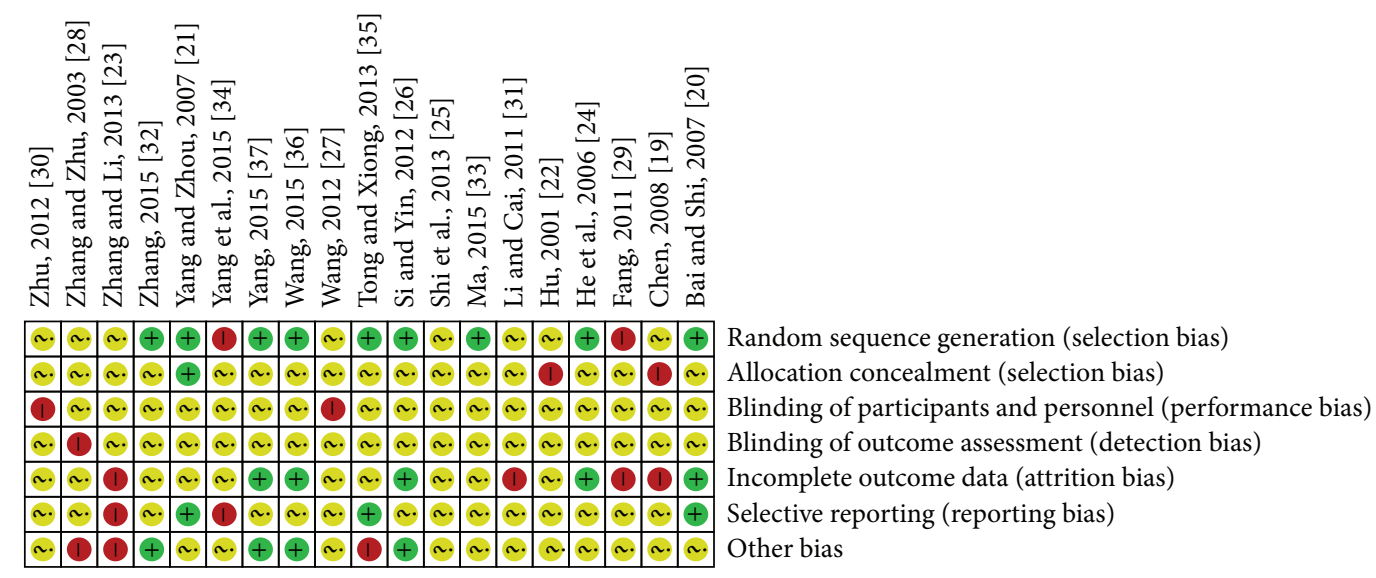

FIGURE 2: Risk of bias summary: reviewing authors' judgments about each risk of bias item for each included study.

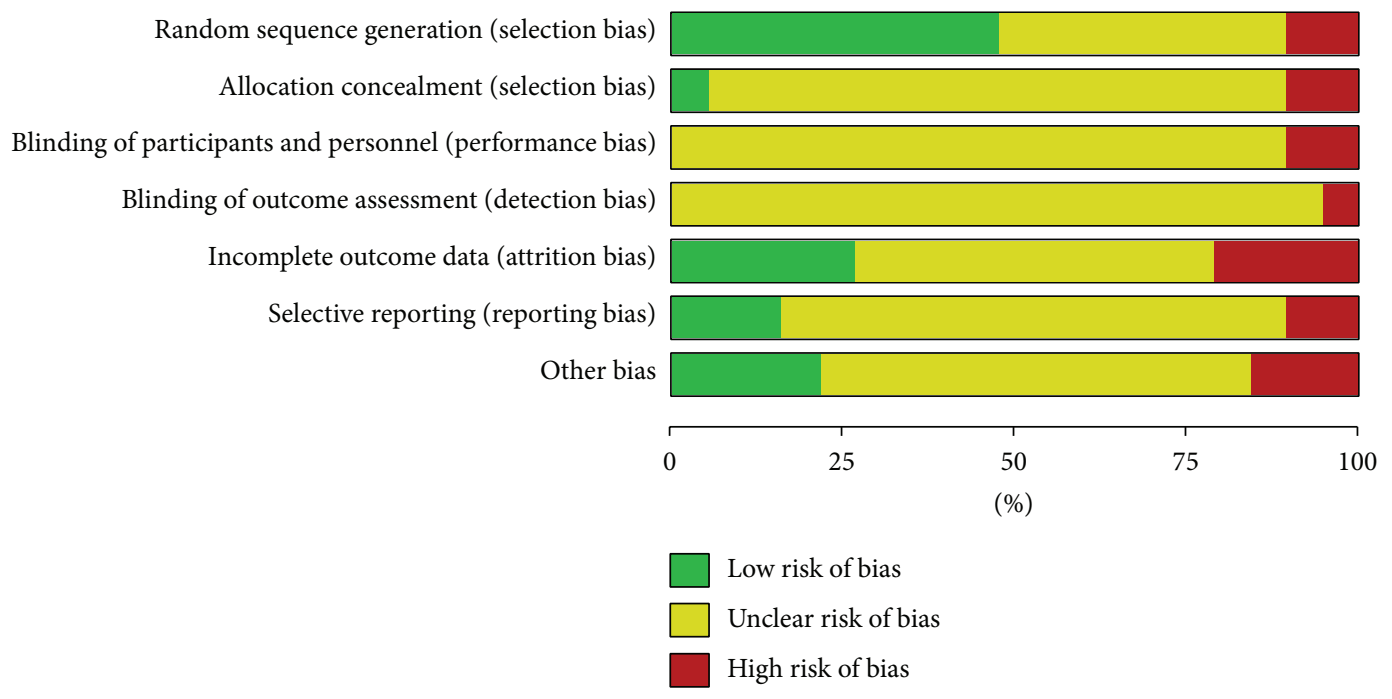

FIGURE 3: Risk of bias graph: reviewing authors' judgments about each risk of bias item presented as percentages across all included studies.

as no preregistered protocols could be obtained from the primary authors. The details of the risk of bias of each trial are presented in Figures 2 and 3.

3.4. Effects of the Interventions. There was no report of mortality as the primary outcome measures (e.g., AMI, severity arrhythmia, heart failure, and revascularization). We analyzed the outcomes, RAS (19 trials), ECG (13 trials), and blood lipid (HDL-C, LDL-C) level (4 trials), and used subgroup analysis with consideration of clinical heterogeneity across the studies. 19 studies were divided into two groups: one evaluated the effects of GLXBBX versus conventional drugs; the other compared GLXBBX plus conventional drugs versus conventional drugs alone.

3.4.1. Reduction of Angina Symptoms (RAS). All the 19 trials [19-37] reported the RAS for AP. Six trials [19-24] compared GLXBBX with conventional drugs. Homogeneity in the results is shown $\left(\chi^{2}=1.39\right.$, $\left.\mathrm{df}=5(P=0.93), I^{2}=0 \%\right)$. Thus, we did a quantitative data synthesis (meta-analysis) by fixed effects model. The meta-analysis showed that there is significant beneficial effect on the GLXBBX group compared to conventional drugs using alone (RR: 1.24 , 95\% CI 1.14 to 1.35; $P<0.00001$ ) (Figure 4). The improvement of RAS was reported in 13 RCTs [25-37] involving 1237 participants and results favored GLXBBX combined with conventional drugs (RR: 1.17 [1.12, 1.22]; $P<0.00001)$ without significant heterogeneity $\left(\chi^{2}=7.03, \mathrm{df}=12, I^{2}=0 \%\right)$ (Figure 5).

3.4.2. ECG Improvement. $13 \mathrm{RCT}$ evaluated the effect of ECG improvement $[19,21-28,32-34,36]$. ECG was significantly improved in the GLXBBX group when compared with conventional drugs (RR: $1.28[1.13,1.44] ; P<0.0001$, Figure 6), with no significant heterogeneity $\left(\chi^{2}=1.98, P=0.74 ; I^{2}=\right.$ $0 \%)$. After analyzing 8 RCTs [25-28, 32-34, 36] involving 868 participants, the result also indicated favoring GLXBBX combined with conventional drugs group ( $\mathrm{RR}=1.22 ; 95 \% \mathrm{CI}$ $=[1.14,1.30] ; P<0.00001)$ in the improvement of ECG and with significant homogeneity $\left(\chi^{2}=4.09 ; I^{2}=0 \%\right)$. The effect estimates were shown in Figure 7. 


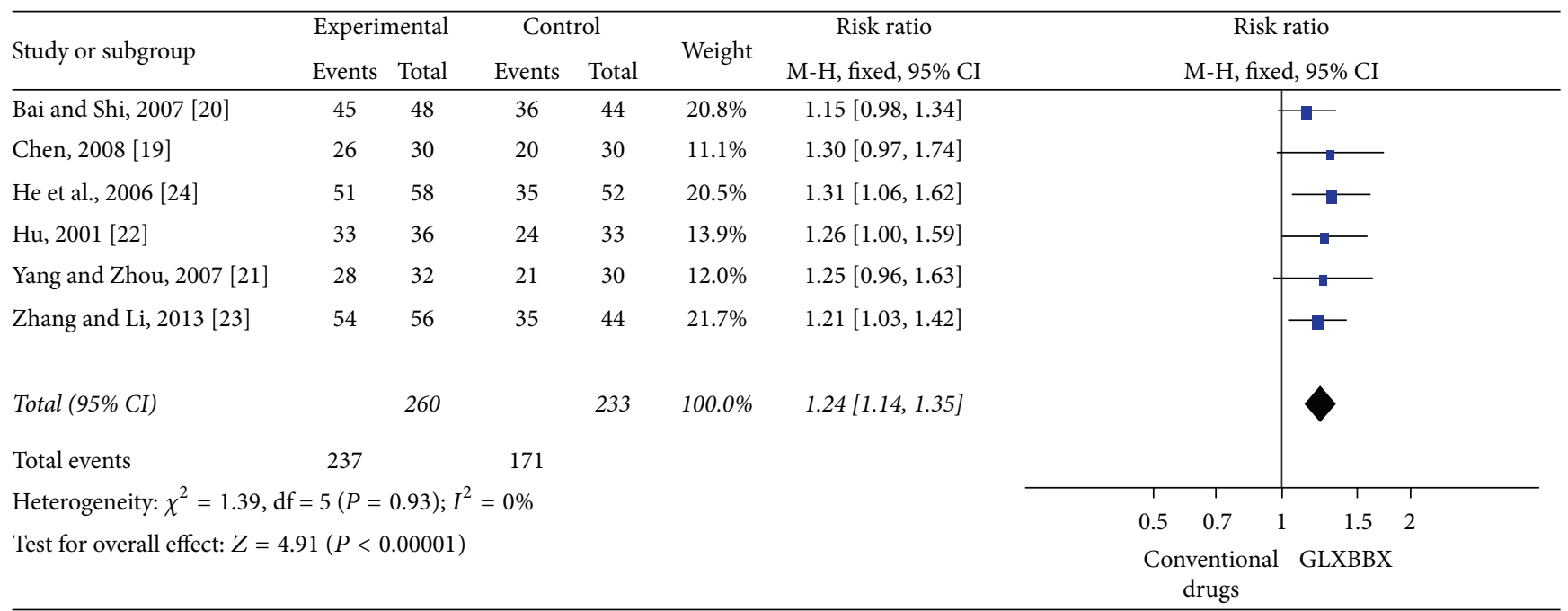

FIGURE 4: Analysis of RAS for AP. Forest plot of comparison: GLXBBX versus conventional drugs.

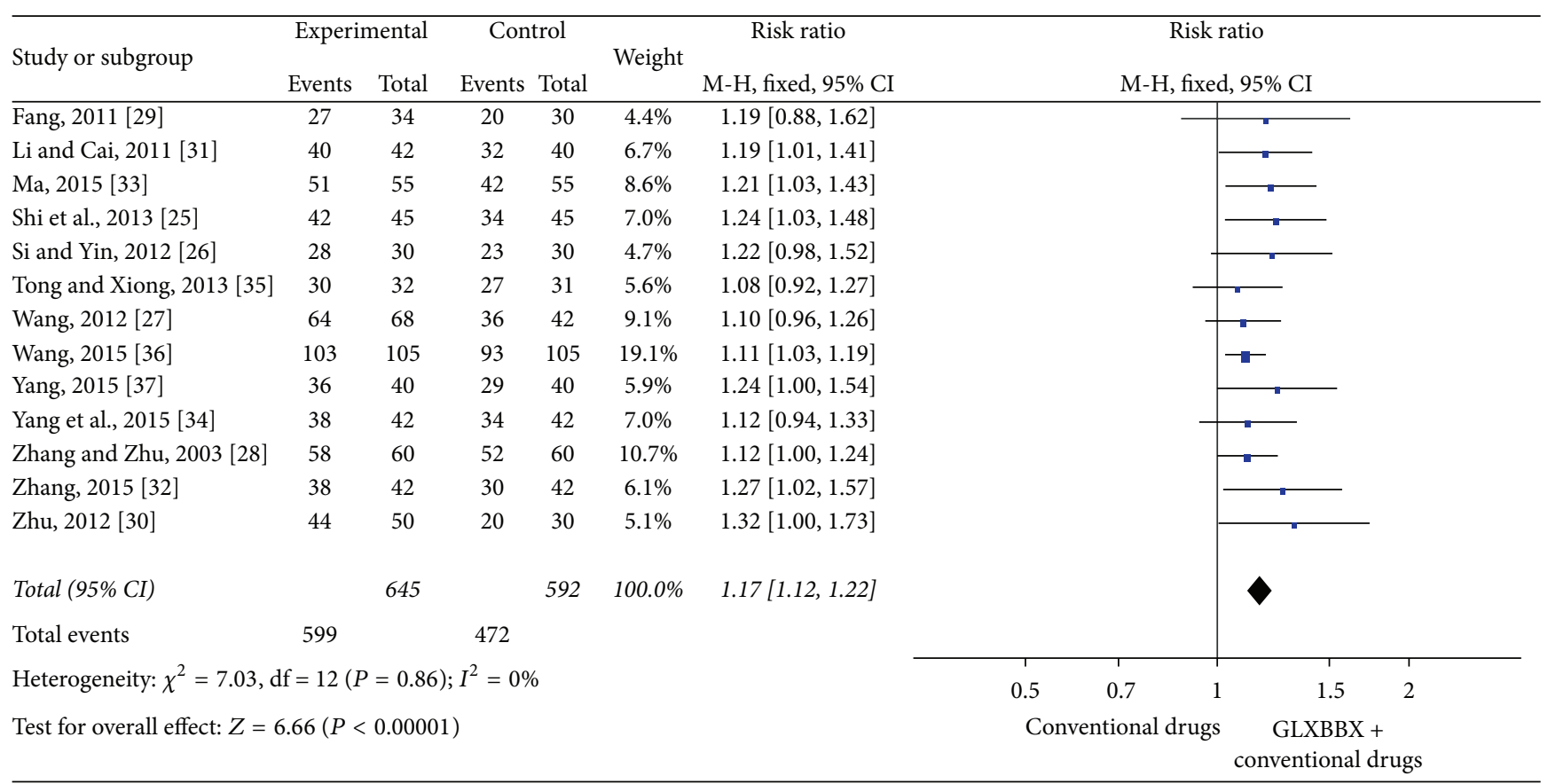

FIgURE 5: Analysis of RAS for AP. Forest plot of comparison: GLXBBX combined with conventional drugs versus conventional drugs.

3.4.3. Other Outcomes (Frequency of Angina Attack and Blood Lipid). Compared with conventional medicine, one trial [27] indicated that frequency of angina attack decreased significantly $(P<0.05)$ in GLXBBX plus conventional drugs group. Two trials $[23,24]$ reported the improvement of HDL$\mathrm{C}$ of 210 patients after 4 weeks of treatment with GLXBBX alone. The statistical data show that GLXBBX was better than conventional drugs alone (MD: $0.56[0.54,0.58])$ with significant homogeneity $\left(\chi^{2}=1.05 ; I^{2}=5 \%\right)$. The effect estimates were shown in Figure 8.

Compared with conventional drugs, 2 RCTs [23, 24] reported the decline levels of LDL-C after treatment favored
GLXBBX. LDL-C was significantly decreased in GLXBBX but with significant heterogeneity $\left(P=0.001, I^{2}=91 \%\right)$. Thus, we did not adopt a meta-analysis. Zhang and Li [23] indicated that duration of angina attack which decreased from $3.52 \pm$ $1.31 \mathrm{mmol} / \mathrm{L}$ to $1.53 \pm 0.75 \mathrm{mmol} / \mathrm{L}$ after treatment favored GLXBBX. One trial [24] showed that after treatment the level of LDL-C decreased significantly $(P<0.05)$ in GLXBBX group compared to conventional drugs.

Compared with conventional medicine, two individual trials [29, 35] reporting LDL-C after treatment favored GLXBBX plus conventional medicine. No homogeneity in the results is shown $\left(P<0.00001, I^{2}=96 \%\right)$. Thus, we did not 


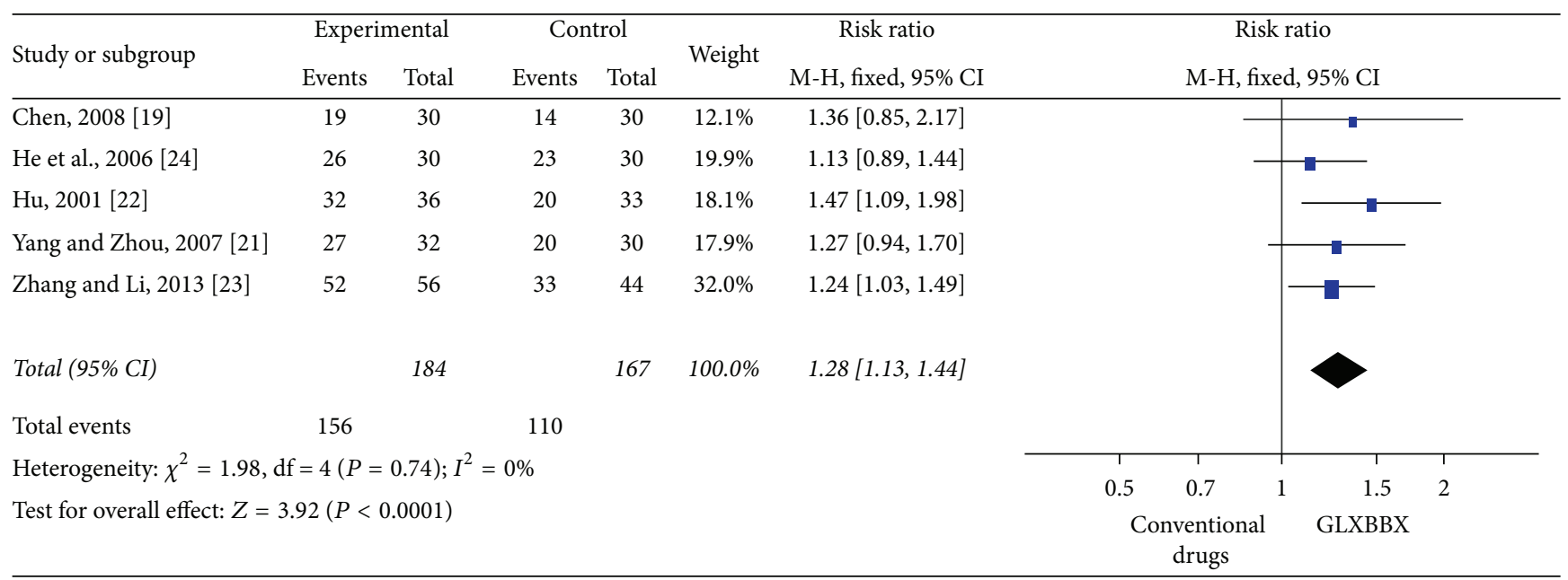

FIGURE 6: Analysis of ECG improvement for AP. Forest plot of comparison: GLXBBX versus conventional drugs.

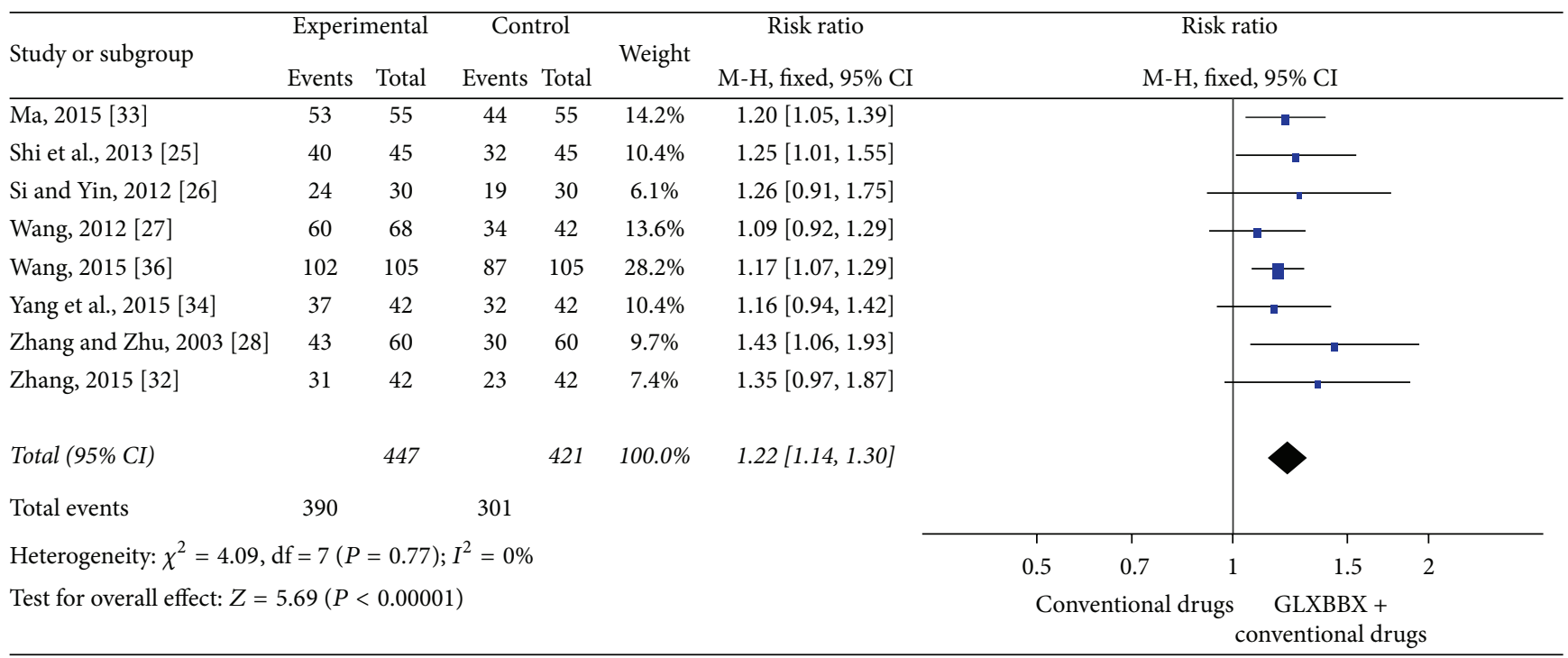

FIGURE 7: Analysis of ECG improvement for AP. Forest plot of comparison: GLXBBX combined with conventional drugs versus conventional drugs.

\begin{tabular}{|c|c|c|c|c|c|c|c|c|c|c|c|c|c|}
\hline \multirow{3}{*}{$\begin{array}{l}\text { Study or subgroup } \\
\text { He et al., } 2006 \text { [24] }\end{array}$} & \multicolumn{3}{|c|}{ Experimental } & \multicolumn{3}{|c|}{ Control } & \multirow{2}{*}{ Weight } & Mean difference & \multirow{2}{*}{\multicolumn{5}{|c|}{$\begin{array}{l}\text { Mean difference } \\
\text { IV, fixed, 95\% CI }\end{array}$}} \\
\hline & \multirow{2}{*}{$\begin{array}{c}\text { Mean } \\
1.74\end{array}$} & \multirow{2}{*}{$\frac{S D}{0.44}$} & \multirow{2}{*}{$\begin{array}{c}\text { Total } \\
58\end{array}$} & \multirow{2}{*}{$\begin{array}{c}\text { Mean } \\
1.27\end{array}$} & \multirow{2}{*}{$\begin{array}{c}\text { SD } \\
0.47\end{array}$} & \multirow{2}{*}{$\begin{array}{c}\text { Total } \\
52\end{array}$} & & \multirow{2}{*}{$\frac{\mathrm{IV} \text {, fixed, } 95 \% \mathrm{CI}}{0.47[0.30,0.64]}$} & & & & & \\
\hline & & & & & & & $1.6 \%$ & & & & & 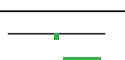 & \\
\hline Zhang and Li, 2013 [23] & 0.63 & 0.07 & 56 & 0.07 & 0.04 & 44 & $98.4 \%$ & $0.56[0.54,0.58]$ & & & & & \\
\hline \multicolumn{3}{|l|}{ Total (95\% CI) } & \multicolumn{2}{|l|}{114} & & 96 & $100.0 \%$ & $0.56[0.54,0.58]$ & & & & 1 & \\
\hline \multicolumn{9}{|c|}{ Heterogeneity: $\chi^{2}=1.05, \mathrm{df}=1(P=0.31) ; I^{2}=5 \%$} & -1 & $\begin{array}{r}1 \\
-0.5\end{array}$ & 0 & 0.5 & 1 \\
\hline \multicolumn{9}{|c|}{ Test for overall effect: $Z=50.60(P<0.00001)$} & & ntiona & & GLXBBX & \\
\hline
\end{tabular}

FIGURE 8: Forest plot of comparison: GLXBBX versus conventional drugs, outcome: HDL-C.

adopt a meta-analysis. Fang [29] indicated that LDL-C which decreased from $3.74 \pm 0.85 \mathrm{mmol} / \mathrm{L}$ to $1.92 \pm 0.67 \mathrm{mmol} / \mathrm{L}$ after treatment favored GLXBBX plus conventional medicine.
Another trial [35] also indicated after treatment favored GLXBBX plus conventional medicine: LDL-C decreased from $4.65 \pm 1.24 \mathrm{mmol} / \mathrm{L}$ to $2.56 \pm 0.72 \mathrm{mmol} / \mathrm{L}$. 
TABLE 3: The effect of GLXBBX for SAP or UAP group, outcome = RAS.

\begin{tabular}{|c|c|c|c|c|c|}
\hline \multirow{2}{*}{ Study ID } & \multicolumn{2}{|c|}{ Response rate\% (response/N) } & \multirow{2}{*}{ Therapeutic gain, $\%$} & \multirow{2}{*}{ NNT } & \multirow{2}{*}{$\mathrm{RR}$} \\
\hline & Experimental & Control & & & \\
\hline Shi et al., 2013 [25] & $93.33(42 / 45)$ & $75.56(34 / 45)$ & 17.77 & 5.63 & 1.24 \\
\hline Wang, 2012 [27] & $94.12(64 / 68)$ & $85.71(36 / 42)$ & 8.41 & 11.89 & 1.10 \\
\hline Zhu, 2012 [30] & $88.00(44 / 50)$ & $66.67(20 / 30)$ & 21.33 & 4.69 & 1.32 \\
\hline $\mathrm{Li}$ and Cai, 2011 [31] & $95.24(40 / 42)$ & $80.00(32 / 40)$ & 15.24 & 6.56 & 1.19 \\
\hline Zhang, 2015 [32] & $90.48(38 / 42)$ & $71.43(30 / 42)$ & 19.05 & 5.25 & 1.27 \\
\hline Ma, 2015 [33] & $92.73(51 / 55)$ & $76.36(42 / 55)$ & 16.37 & 6.11 & 1.21 \\
\hline Yang et al., 2015 [34] & $90.48(38 / 42)$ & $80.95(34 / 42)$ & 9.53 & 10.49 & 1.12 \\
\hline Yang, 2015 [37] & $90.00(36 / 40)$ & $72.50(29 / 40)$ & 17.5 & 5.71 & 1.24 \\
\hline Pooled RR & $91.93(353 / 384)$ & $76.49(257 / 336)$ & 15.44 & 6.48 & 1.20 \\
\hline
\end{tabular}

TABLE 4: The effect of GLXBBX for SAP or UAP group, outcome = ECG.

\begin{tabular}{|c|c|c|c|c|c|}
\hline \multirow{2}{*}{ Study ID } & \multicolumn{2}{|c|}{ Response rate\% (response/N) } & \multirow{2}{*}{ Therapeutic gain, $\%$} & \multirow{2}{*}{ NNT } & \multirow{2}{*}{$\mathrm{RR}$} \\
\hline & Experimental & Control & & & \\
\hline Shi et al., 2013 [25] & $88.89(40 / 45)$ & $71.11(32 / 45)$ & 17.78 & 5.62 & 1.25 \\
\hline Zhang, 2015 [32] & $73.81(31 / 42)$ & $54.76(23 / 42)$ & 19.05 & 5.25 & 1.35 \\
\hline $\mathrm{Ma}, 2015$ [33] & $96.36(53 / 55)$ & $80.00(44 / 55)$ & 16.36 & 6.11 & 1.20 \\
\hline Yang et al., 2015 [34] & $88.10(37 / 42)$ & $76.19(32 / 42)$ & 11.90 & 8.40 & 1.16 \\
\hline Pooled RR & $87.5(161 / 184)$ & $71.20(131 / 184)$ & 16.30 & 6.13 & 1.23 \\
\hline
\end{tabular}

TABLE 5: The effect of GLXBBX for SAP, outcome = RAS.

\begin{tabular}{lcccc}
\hline \multirow{2}{*}{ Study ID } & \multicolumn{2}{c}{ Response rate\% (response/N) } & \multirow{2}{*}{ Therapeutic gain, \% } & \multirow{2}{*}{ NNT } \\
& Experimental & Control & 10.00 & 10.00 \\
Zhang and Zhu, 2003 [28] & $96.67(58 / 60)$ & $86.67(52 / 60)$ & 12.74 & 1.12 \\
Fang, 2011 [29] & $79.41(27 / 34)$ & $66.67(20 / 30)$ & 9.53 & 1.19 \\
Wang, 2015 [36] & $98.10(103 / 105)$ & $88.57(93 / 105)$ & 9.85 & 10.49 \\
Pooled RR & $94.47(188 / 199)$ & $84.62(165 / 195)$ & 10.15 \\
\hline
\end{tabular}

TABLE 6: The effect of GLXBBX for SAP, outcome = ECG.

\begin{tabular}{lccrr}
\hline \multirow{2}{*}{ Study ID } & \multicolumn{2}{c}{ Response rate\% (response/N) } & Therapeutic gain, \% & NNT \\
& Experimental & Control & RR \\
\hline Zhang and Zhu, 2003 [28] & $71.67(43 / 60)$ & $50.00(30 / 60)$ & 21.67 & 4.61 \\
Wang, 2015 [36] & $97.14(102 / 105)$ & $82.86(87 / 105)$ & 14.28 & 7.43 \\
Pooled RR & $87.88(145 / 165)$ & $70.91(117 / 165)$ & 16.98 & 1.17 \\
\hline
\end{tabular}

3.5. Subgroup Analysis. Three subgroups were analyzed based on methodological variables of different AP subtypes; 13 studies provided the data necessary to perform our evaluation. Of these RCTs, 2 RCTs $[26,35]$ involved participants with UAP, 3 RCTs $[28,29,36]$ involved participants with SAP, and the other 8 RCTs $[25,27,30-34,37]$ involved participants with SAP or UAP. Overall, (1) for patients with diagnosis of either SAP or UAP, 91.93\% reported RAS improvement in the experimental group compared with $76.49 \%$ in the control group (therapeutic gain $=15.44 \%$ with an $\mathrm{NNT}=6.48$ ) (Table 3), and ECG improvement was $87.5 \%$ versus $71.20 \%$ (therapeutic gain $=16.30 \%$ with an NNT $=6.13$ ) (Table 4$).(2)$ For patients with SAP, $94.47 \%$ reported RAS improvement after the treatment with GLXBBX plus conventional drugs compared with $84.62 \%$ after the treatment with conventional drugs only (therapeutic gain $=9.85 \%$ with a number needed to treat NNT $=10.15$ ) (Table 5) and $87.88 \%$ compared with $70.91 \%$ in ECG improvement (therapeutic gain $=16.98 \%$ with an NNT = 5.89) (Table 6). (3) For patients with UAP, 93.55\% reported RAS improvement in the experimental group compared with $81.97 \%$ in the control group (therapeutic gain = $11.59 \%$ with an NNT $=8.63)$ (Table 7), and ECG improvement was $80.00 \%$ versus $63.33 \%$ (therapeutic gain $=16.67 \%$ with an NNT $=6.00)($ Table 8$)$.

3.6. Adverse Effect. A total of 8 trials $[19,24,25,27,33,35-$ 37] mentioned the occurrence of adverse effects. The four studies $[19,25,36,37]$ reported that 4 patients with nausea $(4 / 145,2.76 \%)$ were identified in the GLXBBX combined with conventional drugs group, whereas 8 patients with 
TABLE 7: The effect of GLXBBX for UAP, outcome = RAS.

\begin{tabular}{lcccr}
\hline \multirow{2}{*}{ Study ID } & \multicolumn{2}{c}{ Response rate\% (response/N) } & Therapeutic gain, \% & NNT \\
& Experimental & Control & 16.66 & 6.00 \\
\hline Si and Yin, 2012 [26] & $93.33(28 / 30)$ & $76.67(23 / 30)$ & 6.65 & 1.22 \\
Tong and Xiong, 2013 [35] & $93.75(30 / 32)$ & $87.10(27 / 31)$ & 11.59 & 1.04 \\
Pooled RR & $93.55(58 / 62)$ & $81.97(50 / 61)$ & 8.63 \\
\hline
\end{tabular}

TABLE 8: The effect of GLXBBX for UAP, outcome = ECG.

\begin{tabular}{llrrr}
\hline \multirow{2}{*}{ Study ID } & \multicolumn{2}{c}{ Response rate\% (response/N) } & Therapeutic gain, \% & NNT \\
& Experimental & Control & 16.67 & 6.00 \\
Si and Yin, 2012 [26] & $80.00(24 / 30)$ & $63.33(19 / 30)$ & 16.67 & 1.26 \\
Pooled RR & $80.00(24 / 30)$ & $63.33(19 / 30)$ & 6.00 & 1.26 \\
\hline
\end{tabular}

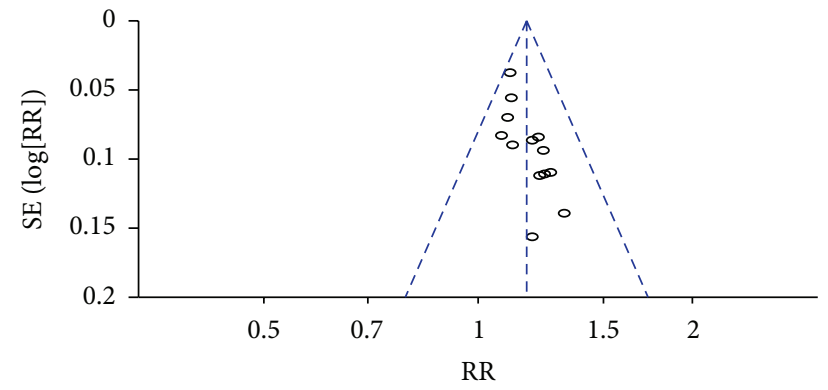

FIGURE 9: Funnel plot of comparison: GLXBBX combined with conventional drugs versus conventional drugs for the outcome of RAS.

gastrointestinal reaction $(8 / 175,4.57 \%)$ and 12 patients with headache $(12 / 115,10.43 \%)$ were observed in the conventional drugs group. And the remaining 4 trials [24, 27, 33, 35] reported that no adverse effects occurred. No severe adverse events were reported.

3.7. Publication Bias. The forest plot of comparison of GLXBBX combined with conventional drugs and conventional drugs for the outcome of RAS was shown in Figure 9.

\section{Discussion}

4.1. Summary of Evidences. Angina pectoris (AP) is a highly prevalent condition in persons with known coronary artery disease (CAD), and the burden of cardiovascular (CV) disease (CVD) remains high, with more than 2200 Americans dying of CVD every day [41, 42]. The aim of management is to abolish or minimise symptoms and to improve quality of life and long-term morbidity and mortality [43]. Most patients improved ECG and symptoms by conventional treatment of western medicine. However, the current status of treatment is unsatisfactory [44]. Therefore, it is very important to seek for more safe and effective prevention and treatment. Recently, with the growing and sustained interest in the benefits of traditional Chinese herbs and integrative medicine, GLXBBX is widely used to treat AP in clinical practice for a long time in China. Meanwhile, there have been a large number of fundamental research and clinical trials of GLXBBX on $\mathrm{AP}$ and RCTs; however, no high level of evidence such as systematic review or meta-analysis was provided for further recommendation. To the best of our knowledge, this is the first SR of GLXBBX D in English.

Nineteen claimed RCTs, with a total of 1730 patients with AP, met the inclusion criteria and were included in this review. The results suggested that RAS and ECG were significantly improved in patients receiving GLXBBX plus conventional drugs therapy or GLXBBX alone. The combination therapy of GLXBBX and conventional drugs could also reduce the frequency of angina attack. In recent years, there are many studies proving the role of lipid profile fractions for the development of coronary artery disease (CAD) $[45,46]$. Previous studies demonstrated that endothelial dysfunction and increased oxidative stress are associated with the dysfunction and dysregulation of individual lipids [47-50]. Some clinical trials and basic researches showed the arterial stiffness correlated positively with specific lipid and oxidative stress and the triacylglycerol (TAG), verylow-density lipoprotein (VLDL), and total cholesterol/highdensity lipoprotein (TC/HDL) were significantly affecting the severity of myocardial damage in the patients of UAP $[51,52]$. Therefore, lowering blood lipid level is one of the important measures to protect the CVD. In this review, GLXBBX can effectively decrease blood LDL-C combined with conventional drugs. Moreover, GLXBBX was found to be effective in terms of improving blood HDL-C level which benefits patients with CVD and decreases blood LDL$\mathrm{C}$ level, when compared with conventional drugs. This result is encouraging which indicates new optional treatment for AP, but the methodological quality of the trials was evaluated generally as low, and the conclusion needs to be confirmed by further study.

4.2. Limitations. Firstly, the following problems reported contribute to the limited methodological quality of most included trials. Although all studies claimed randomization, only 9 studies $[21,24,26,32,33,35-37]$ stated the method of the sequence generation with random number table and drawing [20], and the other 10 trials just mentioned 
"randomly allocating" with no detailed information. In addition, all the trials did not describe allocation concealment in detail and blinding of participants and personnel and blinding of outcome assessment were unclear, which lead to inability to judge whether the study was conducted properly. No multicenter, large-scale RCTs were identified and none of trials had a pretrial estimation of sample size, which indicated the lack of statistical power to ensure appropriate estimation of the therapeutic effect. No trial reported information on follow-up; AP can typically recur across the life span and so long-term follow-up is required for accurate analysis. Only two trials $[33,35]$ had reported dropout with the detailed reasons, which might have led to attrition bias and other biases.

Secondly, heterogeneity is another critical issue that should be considered. The 4 independent trials [23, 24, 29, 35] using GLXBBX alone or combined with conventional drugs compared with conventional drugs did show significant heterogeneity in the results of blood LDL-C level. The significant clinical heterogeneity reflected in variations in methodological quality, participants, interventions, and conventional drugs might weaken the reliability of the data [53]. Four trials specified 3 diagnostic criteria with different types of angina (SAP, UAP) and the durations of treatment were various (ranged from 3 to 8 weeks). In addition, the methodological quality of the trials $[23,24,29,35]$ included was different; 2 RCTs [24, 35] were scored as having superior quality, which mentioned random sequence generation. As a result, the limited number of included trials and different interventions in the GLXBBX and conventional drugs groups restricted us from conducting meaningful subgroup analyses.

Thirdly, none of the included trials reported severe adverse events possibly related to GLXBBX, and the adverse effects included 4 cases with nausea, $2.76 \%$ (4/145), 8 cases with gastrointestinal reaction, $4.57 \%(8 / 175)$, and 12 cases with headache, $10.43 \%$ (12/115), and these side effects may be related to the adverse effect of conventional drugs. Safety is a serious concern that should be recorded in detail. Thus, definitive conclusions about the safety of GLXBBX still cannot be drawn since 4 of 19 trials did report information on safety.

Fourthly, Figure 9 was asymmetrical, which indicated that publication bias might influence the results of our analysis. Moreover, while GLXBBX is a widely used therapy for AP in China, positive results were reported in most of the included studies and some negative results could not be reported, so a certain degree of potential selective bias might exist in this conclusion.

Last but not least, apart from the limitations on the mediocre methodological quality of included studies, the inadequate reporting of mortality or the incidence of complications was another important limitation of this systematic review. Moreover, outcome measures of AP including RAS and ECG improvement and the blood lipid level and frequency of angina attack were limited by relatively small sample size. As a result, evaluating the efficacy of GLXBBX on AP requires more strictly designed large-scale randomized clinical trials.

\section{Conclusion}

From this systematic review, we find that patients receiving GLXBBX adjunct therapy alone or combined with conventional drugs could significantly improve RAS, ECG, the blood lipid level, and frequency of angina attack in patient with AP. But the previous results should be read with caution owing to the poor methodological quality and some possible biases. Thereby, in order to explore the efficacy and safety of GLXBBX treating AP, well-designed, complete efficacy indicator, larger scaled, and multicenter randomized clinical trials with long-term follow-up are warranted for stronger evidence in the future.

\section{Disclosure}

The funders had no role in study design, data collection and analysis, decision to publish, or preparation of the manuscript.

\section{Competing Interests}

All authors declare that they have no conflict of interests.

\section{Authors' Contributions}

Wei Liu, Xingjiang Xiong, and Xiaochen Yang contributed equally to this paper.

\section{Acknowledgments}

The current work was partially supported by the National Natural Science Foundation of China (no. 81273741), Capital Clinical Medicine Application Characteristic Project of Beijing Science and Technology Committee (no. Z131107002213152), Scientific Research Common Program of Beijing Municipal Commission of Education (no. KM201311420084), and Basic Clinical Cooperation Projects of Capital Medical University (no. 13JL86).

\section{References}

[1] J.-C. Tardif, "Coronary artery disease in 2010," European Heart Journal, vol. 12, pp. C2-C10, 2010.

[2] E. Braunwald and D. A. Morrow, "Unstable angina: is it time for a requiem?” Circulation, vol. 127, no. 24, pp. 2452-2457, 2013.

[3] J. Zarifis, V. Grammatikou, M. Kallistratos, and A. Katsivas, "Treatment of stable angina pectoris with ivabradine in everyday practice: a pan-hellenic, prospective, noninterventional study," Clinical Cardiology, vol. 38, no. 12, pp. 725-732, 2015.

[4] GBD 2013 Mortality and Causes of Death Collaborators, "Global, regional, and national age-sex specific all-cause and cause-specific mortality for 240 causes of death, 1990-2013: a systematic analysis for the Global Burden of Disease Study 2013," The Lancet, vol. 385, no. 9963, pp. 117-171, 2015.

[5] J. S. Kanu, Y. Gu, S. Zhi et al., "Single nucleotide polymorphism rs3774261 in the AdipoQ gene is associated with the risk of coronary heart disease (CHD) in Northeast Han Chinese population: a case-control study," Lipids in Health and Disease, vol. 15, no. 1, article 6, 2016. 
[6] D. M. Lloyd-Jones, Y. Hong, D. Labarthe et al., "Defining and setting national goals for cardiovascular health promotion and disease reduction: the American Heart Association's strategic impact goal through 2020 and beyond," Circulation, vol. 121, no. 4, pp. 586-613, 2010.

[7] C. A. Daly, B. De Stavola, and K. M. Fox, "Predicting prognosis in stable angina-results from the Euro heart survey of stable angina: Prospective observational study," The British Medical Journal, vol. 332, no. 7536, pp. 262-265, 2006.

[8] K. Fox, M. A. Alonso Garcia, D. Ardissino et al., "Guidelines on the management of stable angina pectoris: Executive summary: the Task Force on the Management of Stable Angina Pectoris of the European Society of Cardiology," European Heart Journal, vol. 27, no. 11, pp. 1341-1381, 2006.

[9] J. F. Beltrame, A. J. Weekes, C. Morgan, R. Tavella, and J. A. Spertus, "The prevalence of weekly angina among patients with chronic stable angina in primary care practices," Archives of Internal Medicine, vol. 169, no. 16, pp. 1491-1499, 2009.

[10] M. A. Hlatky, D. B. Boothroyd, K. A. Melsop et al., "Medical costs and quality of life 10 to 12 years after randomization to angioplasty or bypass surgery for multivessel coronary artery disease," Circulation, vol. 110, no. 14, pp. 1960-1966, 2004.

[11] J. M. Tarkin and J. C. Kaski, "Pharmacological treatment of chronic stable angina pectoris," Clinical Medicine, vol. 13, no. 1, pp. 63-70, 2013.

[12] Y. Wee, K. Burns, and N. Bett, "Medical management of chronic stable angina," Australian Prescriber, vol. 38, no. 4, pp. 131-136, 2015.

[13] T. Münzel and T. Gori, "Nitrate therapy and nitrate tolerance in patients with coronary artery disease," Current Opinion in Pharmacology, vol. 13, no. 2, pp. 251-259, 2013.

[14] Y. Ren, D. Li, H. Zheng et al., "Acupoint application in patients with chronic stable angina pectoris: study protocol of a randomized, double-blind, controlled trial," Evidence-Based Complementary and Alternative Medicine, vol. 2014, Article ID 619706, 8 pages, 2014.

[15] Y.-F. Ding, Y.-R. Peng, J. Li, H. Shen, M.-Q. Shen, and T.-H. Fang, "Gualou Xiebai Decoction prevents myocardial fibrosis by blocking TGF-beta/Smad signalling," Journal of Pharmacy and Pharmacology, vol. 65, no. 9, pp. 1373-1381, 2013.

[16] H.-M. Zhang, D.-L. Tang, L. Tong et al., "Gualou Xiebai Banxia Decoction inhibits NF-kappa B-dependent inflammation in myocardial ischemia-reperfusion injury in rats," Journal of Traditional Chinese Medicine, vol. 31, no. 4, pp. 338-343, 2011.

[17] X. J. He, N. L. Wang, F. Qiu, and X. S. Yao, "Study on the active spirostanol saponins of Gualou xiebai baijiutang," Yaо Xue Xue Bao, vol. 38, no. 6, pp. 433-437, 2003.

[18] H.-Y. Yan, C.-C. Zou, M.-L. Wei, Z.-Z. Gao, and Y. Yang, "Pharmacodynamics Study on gualou xiebai dropping pills and its medicinal ingredients in prescription," Zhong Yao Cai, vol. 38, no. 3, pp. 567-571, 2015.

[19] G. X. Chen, "Clinical observation of Gualou xiebai banxia Decoction on angina pectoris," Hubei Journal of Traditional Chinese Medicine, vol. 10, no. 3, pp. 40-42, 2008.

[20] J. M. Bai and W. F. Shi, "Clinical observation and analysis of Gualou xiebai banxia Decoction for 48 cases of angina," Journal of Sichuan of Traditional Chinese Medicine, vol. 25, no. 9, pp. 6465, 2007.

[21] J. D. Yang and D. H. Zhou, "Clinical observation of Gualou xiebai banxia Decoction for 32 cases of angina," JETCM, vol. 16, no. 12, pp. 1530-1532, 2007.
[22] X. F. Hu, "Clinical observation on 36 cases of angina pectoris of coronary heart disease treated by gualou xiebai banxia decoction," Hunan Guiding Journal of TCMP, vol. 7, no. 9, pp. 454-456, 2001.

[23] D. F. Zhang and X. L. Li, "Clinical observation of Gualou xiebai banxia Decoction for 56 cases with angina pectoris of coronary heart disease," Clinical Journal of Traditional Chinese Medicine, vol. 25, no. 9, pp. 770-772, 2013.

[24] Y. H. He, Y. Cai, and S. D. Luo, "Clinical observation on treating 58 cases of angina pectoris of coronary heart disease with gualouxiebaibanxia decoction addition," Guiding Journal of TCM, vol. 12, no. 2, pp. 62-64, 2006.

[25] P. F. Shi, K. Wang, Q. L. Li et al., "Observation of gualou xiebai banxia decoction combined with western medicine treating 45 cases of coronary heart disease angina pectoris," Modern Traditional Chinese Medicine, vol. 33, no. 6, pp. 11-13, 2013.

[26] J. X. Si and Z. J. Yin, "Clinical observation of flavored gualou xiebai and banxia decoction in the treatment of unstable angina of coronary heart disease," China Journal of Chinese Medicine, vol. 27, no. 172, pp. 1101-1103, 2012.

[27] Z. Z. Wang, "Clinical observation on 68 cases of angina pectoris of coronary heart disease treated by gualou xiebai banxia decoction,” CJGMCM, vol. 27, no. 5, pp. 927-929, 2012.

[28] Z. Q. Zhang and W. Z. Zhu, "Clinical observation of Gualou xiebai banxia decoction for 60 cases with angina pectoris of coronary heart disease," Zhejiang JITCWM, vol. 13, no. 13, pp. 248-249, 2003.

[29] H. B. Fang, "Observation of gualou xiebai banxia decoction combined with western medicine treating 34 cases of coronary heart disease angina pectoris," Zhejiang Journal of Traditional Chinese Medicine, vol. 46, no. 6, pp. 418-420, 2011.

[30] B. G. Zhu, "Clinical observation and analysis of Gualou xiebai banxia Decoction for 80 cases with angina pectoris of coronary heart disease," CJGMCM, vol. 27, no. 6, pp. 1256-1257, 2012.

[31] J. L. Li and X. B. Cai, "Clinical observation of Flavored Gualou xiebai banxia Decoction for angina pectoris of coronary heart disease," Public Medical Forum Magazine, vol. 15, pp. 846-848, 2011.

[32] H. W. Zhang, "Therapeutic effects of Flavored Gualou xiebai banxia Decoction for 42 patients of angina pectoris of phlegm and blood stasis syndrome," CJGMCM, vol. 30, no. 11, pp. 24392441, 2015.

[33] P. Ma, "Randomized parallel group study Gualou Xiebai soup combined with Western treatment of Angina Pectoris," Journal of Practical Traditional Chinese Internal Medicine, vol. 29, no. 9, pp. 110-112, 2015.

[34] Q. F. Yang, D. F. Bi, H. B. Wu et al., "Clinical observation of Gualouxiebaibanxia Decoction combined with Nitroglycerin Injection for 42 cases with angina pectoris of coronary heart disease," Guide of Chinese Medicine, vol. 13, no. 1, pp. 228-229, 2015.

[35] R. X. Tong and S. Q. Xiong, "Phlegm method for unstable angina plasma ox-LDL levels affect random parallel control study," Journal of Practical Traditional Chinese Internal Medicine, vol. 27, no. 9, pp. 62-64, 2013.

[36] W. Q. Wang, "Clinical observation of Gualou xiebai banxia Decoction for angina pectoris of coronary heart disease," Heibei Medicine, vol. 21, no. 6, pp. 1033-1035, 2015.

[37] F. Q. Yang, "Clinical observation of modified Gualou xiebai banxia Decoction for 80 cases with angina pectoris of coronary heart disease," China Medical Engineering, vol. 23, no. 8, pp. 125127, 2015. 
[38] E. Rapaport, R. Bernard, and E. Corday, "Nomenclature and criteria for diagnosis of ischemic heart disease. Report of the Joint International Society and Federation of Cardiology/World Health Organization Task Force on standardization of clinical nomenclature," Circulation, vol. 59, no. 3, pp. 607-609, 1979.

[39] R. J. Gibbons, J. Abrams, P. C. Deedwania et al., "ACC/AHA practice guidelines-ACC/AHA 2002 guideline update for the management of patients with chronic stable angina task force on practice guidelines (committee to update the 1999 guidelines)," Circulation, vol. 107, no. 1, pp. 149-158, 2002.

[40] J. P. T. Higgins and S. Green, “Corchrane Reviewers' Handbook 5.1.0 [updated March 2011], Review Manager (RevMan) [Computer program]. Version 5.1.0".

[41] V. A. Barnes and D. W. Orme-Johnson, "Prevention and treatment of cardiovascular disease in adolescents and adults through the transcendental meditation program: a research review update," Current Hypertension Reviews, vol. 8, no. 3, pp. 227-242, 2012.

[42] E.-M. Backé, A. Seidler, U. Latza, K. Rossnagel, and B. Schumann, "The role of psychosocial stress at work for the development of cardiovascular diseases: a systematic review," International Archives of Occupational and Environmental Health, vol. 85, no. 1, pp. 67-79, 2012.

[43] G. Kourlaba, C. Vlachopoulos, J. Parissis, J. Kanakakis, G. Gourzoulidis, and N. Maniadakis, "Ranolazine for the symptomatic treatment of patients with chronic angina pectoris in Greece: a cost-utility study," BMC Health Services Research, vol. 15, no. 1, article 566, 2015.

[44] R. Vigen, J. A. Spertus, T. M. Maddox et al., "Hospital-level variation in angina and mortality at 1 year after myocardial infarction: insights from the Translational Research Investigating Underlying Disparities in Acute Myocardial Infarction Patients' Health Status (TRIUMPH) registry," Circulation: Cardiovascular Quality and Outcomes, vol. 7, no. 6, pp. 851-856, 2014.

[45] J. Y. Park, J. K. Paik, O. Y. Kim, J. S. Chae, Y. Jang, and J. H. Lee, "Interactions between the APOA5-1131T > C andthe FEN1 $10154 \mathrm{G}>\mathrm{T}$ polymorphisms on $\omega 6$ polyunsaturatedfatty acids in serum phospholipids and coronary artery disease," The Journal of Lipid Research, vol. 51, no. 11, pp. 3281-3288, 2010.

[46] D. Kong, Y. Wang, Y. Liu et al., “The association between blood lipid and phlegm turbidity syndrome of angina pectoris: a systematic review and meta-analysis," Complementary Therapies in Medicine, vol. 22, no. 4, pp. 801-813, 2014.

[47] P. Mata, R. Alonso, A. Lopez-Farre et al., "Effect of dietary fat saturation on LDL oxidation and monocyte adhesion to human endothelial cells in vitro," Arteriosclerosis, Thrombosis, and Vascular Biology, vol. 16, no. 11, pp. 1347-1355, 1996.

[48] M. Toborek, Y. W. Lee, R. Garrido, S. Kaiser, and B. Hennig, "Unsaturated fatty acids selectively induce an inflammatory environment in human endothelial cell," The American Journal of Clinical Nutrition, vol. 75, no. 1, pp. 119-125, 2002.

[49] M. Toborek, S. W. Barger, M. P. Mattson, S. Barve, C. J. McClain, and B. Hennig, "Linoleic acid and TNF- $\alpha$ cross-amplify oxidative injury and dysfunction of endothelial cells," Journal of Lipid Research, vol. 37, no. 1, pp. 123-135, 1996.

[50] C. Y. Ha, J. Y. Kim, J. K. Paik et al., “The association of specific metabolites of lipid metabolism with markers of oxidative stress, inflammation and arterial stiffness in men with newly diagnosed type 2 diabetes," Clinical Endocrinology, vol. 76, no. 5, pp. 674-682, 2012.
[51] J. Y. Park, S.-H. Lee, M.-J. Shin, and G.-S. Hwang, "Alteration in metabolic signature and lipid metabolism in patients with angina pectoris and myocardial infarction," PLOS ONE, vol. 10, no. 8, Article ID e0135228, 2015.

[52] K. R. Bagale, A. S. Ingle, and R. Choudhary, "Contribution of various lipid profile parameters in determining creatine kinaseMB levels in unstable angina patients," International Journal of Applied and Basic Medical Research, vol. 6, no. 2, pp. 106-110, 2016.

[53] X. Xiong, P. Wang, X. Li, and Y. Zhang, "Qigong for hypertension: a systematic review," Medicine, vol. 94, no. 1, pp. 1-14, 2015. 


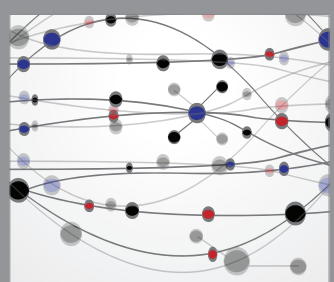

The Scientific World Journal
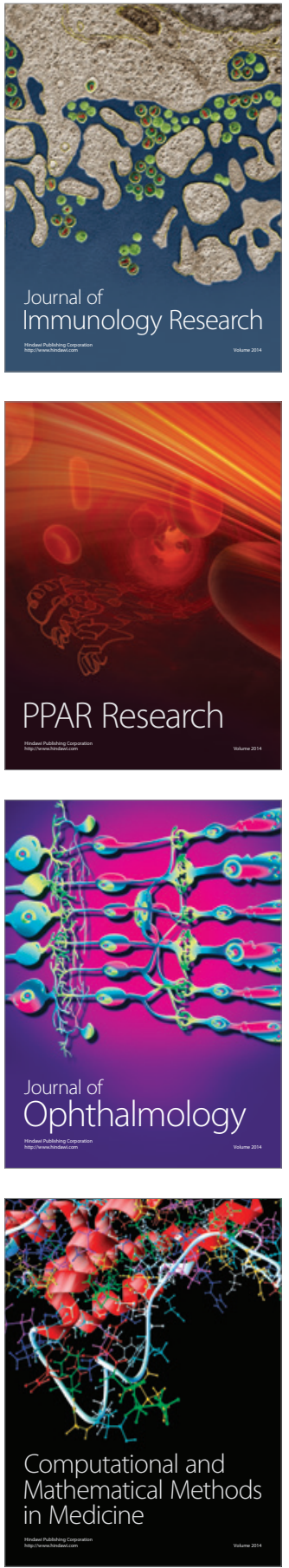

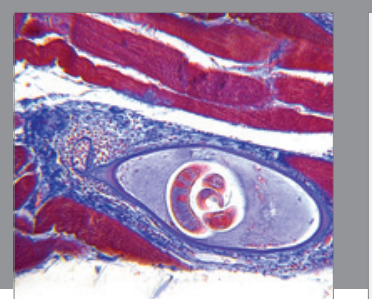

Gastroenterology Research and Practice

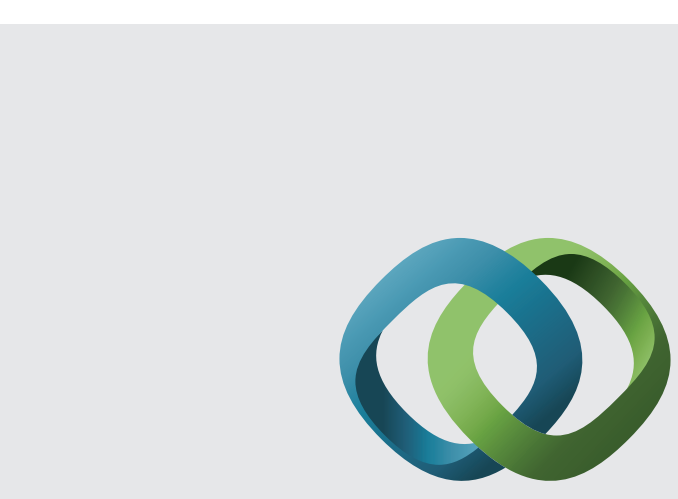

\section{Hindawi}

Submit your manuscripts at

http://www.hindawi.com
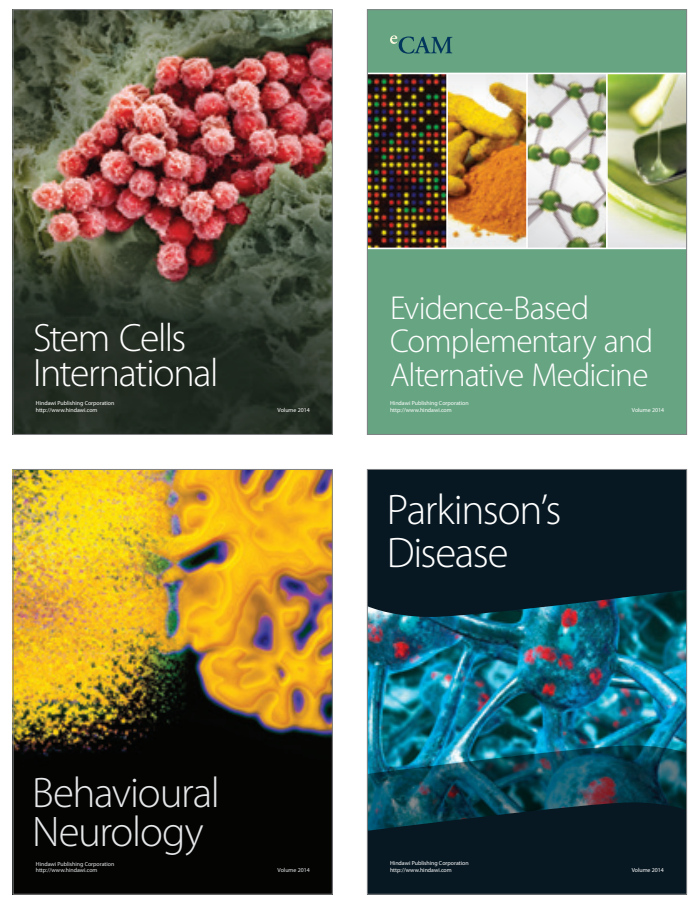
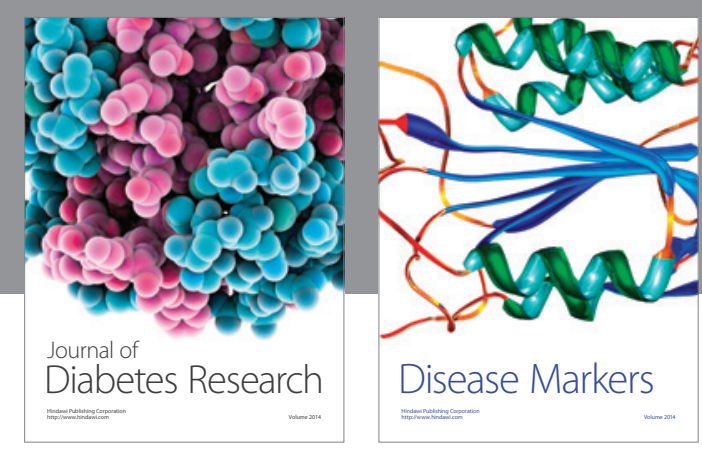

Disease Markers
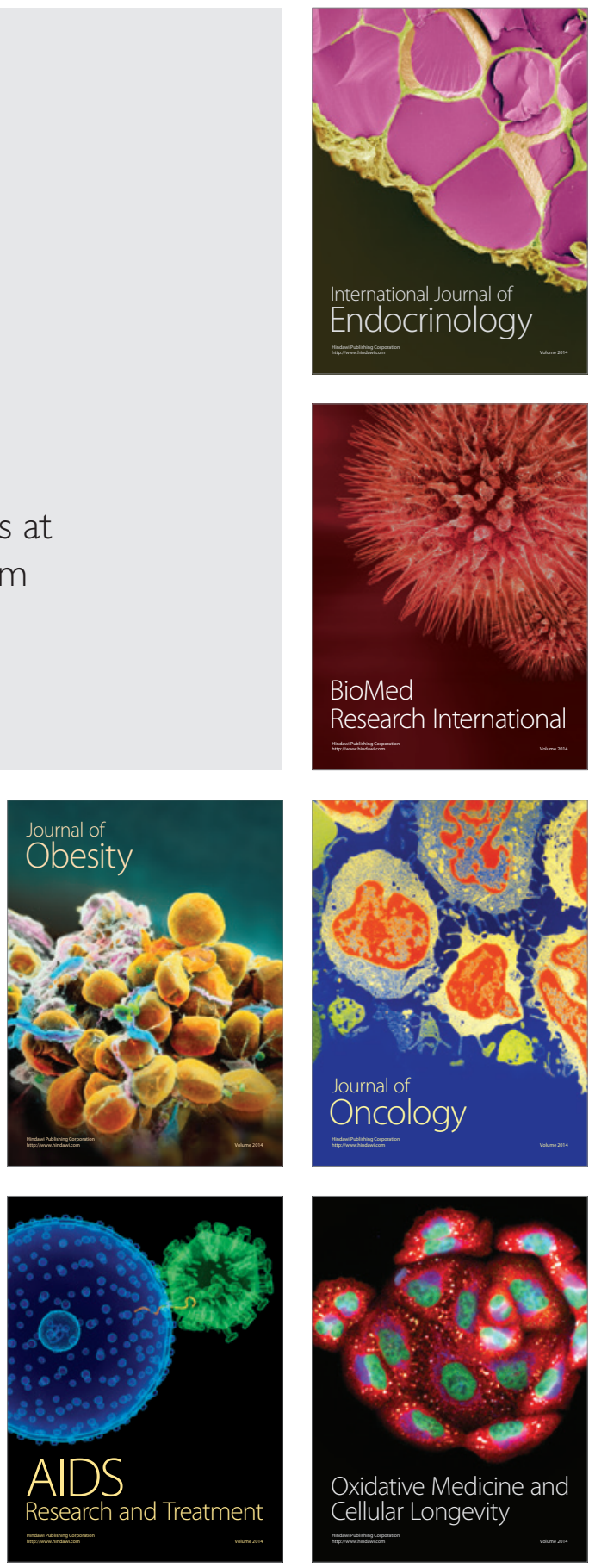